\title{
Study on higher order azimuthally polarized Laguerre-Gaussian mode beams with high NA lens
}

\author{
K. Lalithambigai · P. M. Anbarasan • \\ K. B. Rajesh
}

Received: 1 March 2014 / Accepted: 28 May 2014/Published online: 19 June 2014

(C) The Author(s) 2014. This article is published with open access at Springerlink.com

\begin{abstract}
The intensity distributions near the focal point for tight focusing of azimuthally polarized LaguerreGaussian beam including higher order modes are calculated based on vector diffraction theory. For higher order as well as the fundamental lower order mode $\mathrm{A}-\mathrm{TEM}_{01}{ }^{*}$, a small focal hole at the centre is obtained for the focusing under high NA lens. In a limit of NA $=0.95$, the higher order A-TEM $\mathrm{p}_{1}$ * mode beams of $p=1,2,3,4,5$ and 6 are established numerically in detail. It is observed that one of the higher order mode $\mathrm{A}-\mathrm{TEM}_{51}$ * generates some interesting results. And it is also observed that the intensity distribution of the different mode has little variation among the degree of truncation $(\beta)$ of the input beam beside the pupil. This work is important for optical manipulation, optical data storage, micro-machines and optical trapping applications for low refractive index particles.
\end{abstract}

Keywords Vector diffraction theory - High NA lens . Laguerre-Gaussian · Azimuthally polarized beam · Higher order modes

K. Lalithambigai $(\bowtie) \cdot$ P. M. Anbarasan Department of Physics, Periyar University, Salem 636 011, Tamilnadu, India e-mail: lallykesavan@gmail.com

\section{K. B. Rajesh}

Department of Physics, Chikkanna Government Arts College, Tiruppur, Tamilnadu 641 602, India

\section{Introduction}

Generation and application of optical tube beams are one of the very fascinating subjects in optics. It has built up fast in recent years due to the enormous interest. Optical tube beams are determined by broad-ranging applications with guiding and attaching of living cells [1], optical tube beam traps and manipulation of atoms [2], high-resolution fluorescence microscopy [3], etc. Laguerre-Gaussian (LG) beam plays an important role in assuming the size and shape of the dark core in the diffraction pattern $[4,5]$. The intensity distribution of the LG beam bands annular intensity rings by means of dark core that can entrap living cells and micro-particles [6,7]. While focused by a high numerical aperture (NA) system, LG beam perhaps traps low and high refractive index particles simultaneously [8]. Consequently, LG optical tweezers are recognized as "optical vortices" $[9,10]$. Optical tweezers by means of more sophisticated features can be familiar with higher order mode beams.

The focusing beam was considered to be a single-ringshaped, which is frequently indicated as lower order $\mathrm{TEM}_{01}$ * mode beam [11]. Alternatively, the higher order modes referred as $\mathrm{TEM}_{\mathrm{p} 1}$ * beams and $\mathrm{p}$ consists the number of rings. Moreover, a double-ring-shaped beam, which is usually featured as higher order $\mathrm{TEM}_{11}$ * mode beam. Recently, it was experimentally detected directly on or after a laser cavity by a higher order radially polarized mode $\mathrm{R}-\mathrm{TEM}_{11} *$ [12]. In addition, these higher order mode beams may be created with radial polarization by a particular laser cavity designed to oscillate. Additionally, it was theoretically reported that a double-ringshaped radially polarized beam potentially forms a dark spot around the focal point in an exacting focusing condition [13]. One more studied, the higher order mode R-TEM ${ }_{11}$ * (doublering-shaped) radially polarized beams can successfully decrease the focal spot size by $\pi$ phase shift due to the destructive interference between the inner rings as well as the 
Fig. 1 Azimuthally polarized double-ring-shaped beam passes through high NA objective lens

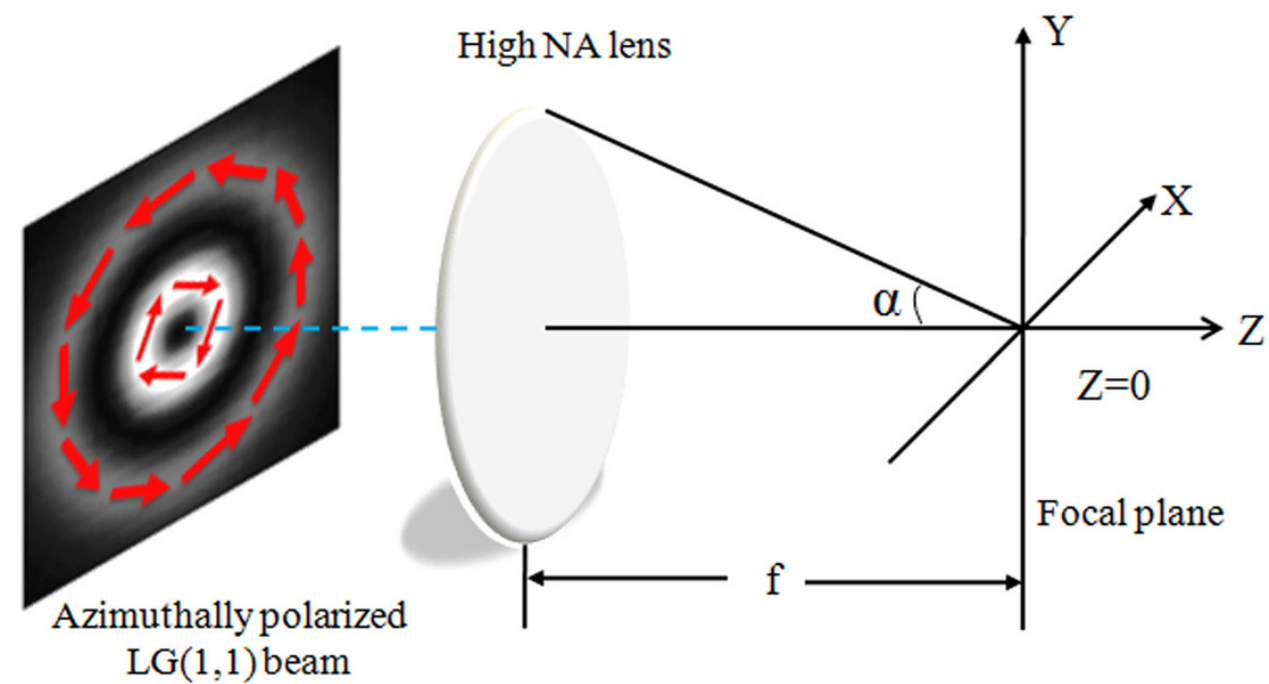

outer rings [14]. More recently, a well created outstanding focal spot, where the spot size, focal depth and the side lobe intensity are $0.41 \lambda, 9.53 \lambda$ and $16.35 \%$ by tight focusing of a higher order radially polarized beam with the 17-belt binary phase pupil filters [15]. In this article, we examine the focusing properties of azimuthally polarized beams together with higher order modes based on vector diffraction theory. For higher order azimuthally polarized modes with a single-ring-shaped A-TEM ${ }_{01} *$ mode beams form a small focal hole at the focal point below the high NA focusing condition. A-TEM A $_{1}$ mode beam can efficiently reduce the focal hole size nearly by $0.324 \lambda$ with focal depth of $3.72 \lambda$ because of the interference between inner and outer rings. Besides the higher order mode A-TEM ${ }_{51} *$ generates some more interesting results and also premeditated the intensity distribution of the different modes has little variation among the degree of truncation $\beta$ of the input beam nearby the pupil.

\section{Theory}

In accordance with Richards and Wolf's vector diffraction method [16] broadly utilized for arbitrary incident polarization with high NA focusing systems and recommended method is shown in Fig. 1. In the case of an incident azimuthally polarization, the electric field $E(r, \varphi, z)$ can be written as [17]:

$$
\begin{aligned}
E(r, \varphi, z) & =\left[\begin{array}{c}
E_{r} \\
E_{\varphi} \\
E_{z}
\end{array}\right] \\
& =\left[\begin{array}{c}
2 A \int_{0}^{\alpha} \cos ^{\frac{1}{2}}(\theta) \sin (\theta) A(\theta) J_{1}(k r \sin \theta) \mathrm{e}^{i k z \cos \theta} \mathrm{d} \theta \\
0
\end{array}\right]
\end{aligned}
$$

where $A$ denotes the relative amplitude, $\alpha$ represents the aperture angle and $\alpha=\operatorname{arc} \sin (\mathrm{NA} / n)$, here NA indicates numerical aperture and $n$ is the index of refraction between the lens and the sample. $J_{1}(x)$ is a symbol of the Bessel functions of the first kind and $A(\theta)$ signifies the amplitude and phase distribution at the exit pupil. For an incident A$\mathrm{TEM}_{\mathrm{p} 1}$ * beam, $A(\theta)$ can be expressed as $[18,19]$ :

$A(\theta)=\beta^{2} \frac{\sin \theta}{\sin ^{2} \theta} \exp \left[-\left(\beta \frac{\sin \theta}{\sin \alpha}\right)^{2}\right] L_{p}^{1}\left[2\left(\beta \frac{\sin \theta}{\sin \alpha}\right)^{2}\right]$

Here, we regard $L_{\mathrm{p}}^{1}$ as generalized Laguerre polynomial and $p+1$ represents the mode number of the incident beam. Truncation coefficient $\beta=R / w$ represents the ratio between $R$ is the radius of the aperture and $w$ is the waist of incident beam. From Fig. $2 \mathrm{a}$, if the mode is taken as $p=0$, the incident azimuthally polarized beam is a single-ringshaped $\mathrm{A}-\mathrm{TEM}_{01} *$ mode azimuthally polarized beam. If the taken modes are larger then one, it comes under the category of higher order azimuthally polarized mode beams at the same time $\beta$ should be take $>1$ under the high NA focusing condition. If $\beta<1$ the external ring of the $\mathrm{A}-\mathrm{TEM}_{\mathrm{p} 1}$ * beam will be entirely blocked beside the pupil. From Fig. 2b, we consider the mode as $p=1$, the incident azimuthally polarized beam is a higher order double-ringshaped $\mathrm{A}-\mathrm{TEM}_{11} *$ mode azimuthally polarized beam. An additional case, if intensity distributions of azimuthally polarized mode is $p=2$; it has triple-ring-shaped and related direct polarization states are shown in Fig. 2c.

\section{Results and discussion}

We carry out the integration of Eq. (1) numerically using factors $\lambda=1$ and NA of the objective is 0.95 . For easy calculations, we presume that the refractive index $n=1$ 
Fig. 2 Bottom Theoretical intensity distributions of azimuthally polarized

A-TEM ${ }_{01}$ * for the mode $p=0$ (single-ring), A-TEM ${ }_{11} *$ for the mode $p=1$ (double-ring),

A-TEM ${ }_{21}$ * for the mode $p=2$ (triple-ring). Top Related polarization states

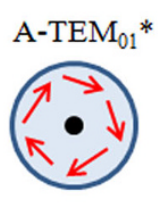

(a)

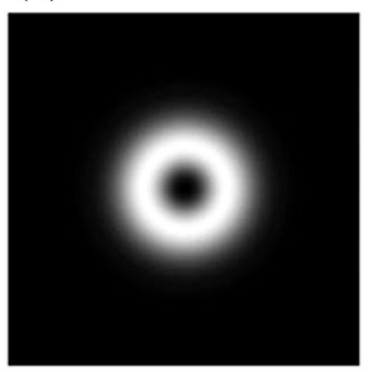

(b)
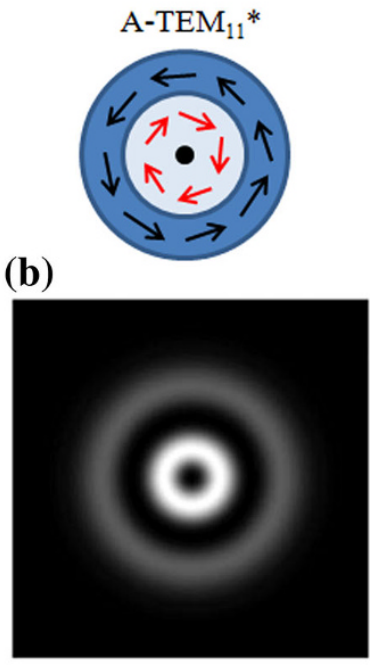

(c)
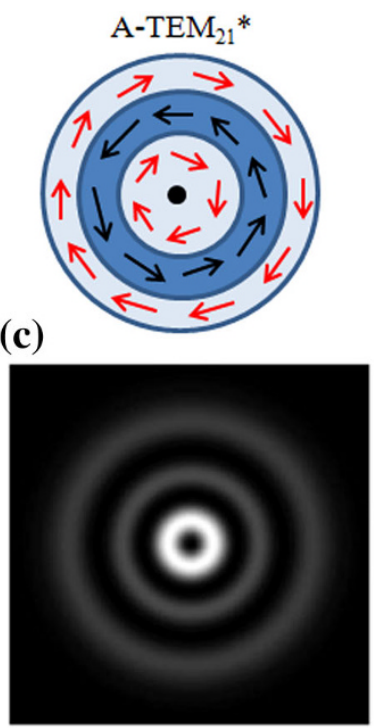

(a)

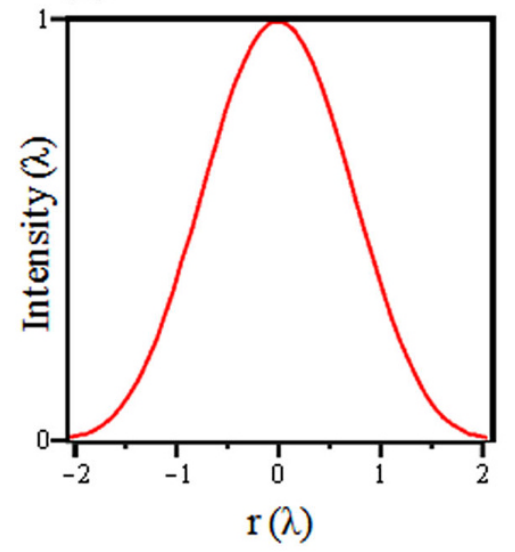

(b)

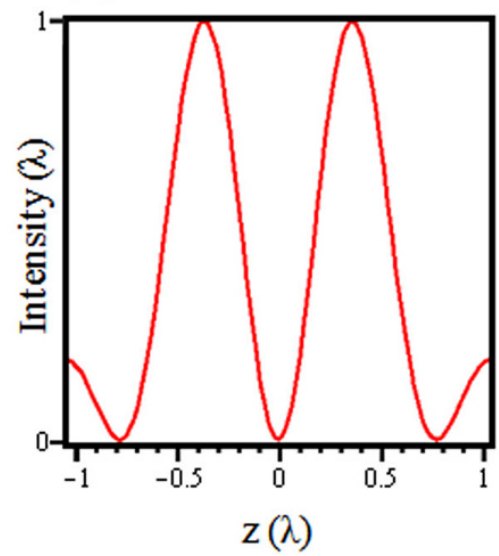

(c)

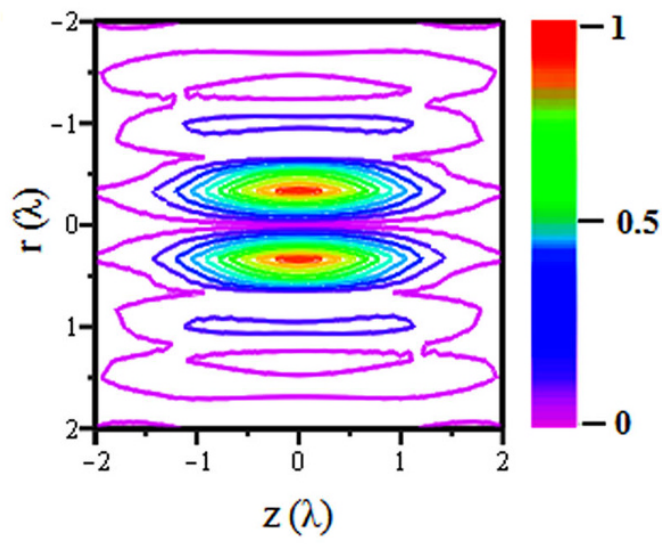

Fig. 3 a Shows intensity distribution of lower order azimuthally polarized A-TEM $01 *$ mode beam focused by high NA lens with corresponding a FWHM, b DOF and c contour profile of the focal hole

and $A=1$. In the subsequent computations, $\beta$ values are determined for each mode beam so that $90 \%$ of the arriving energy focused in the front of the lens. In this effort, we take $\beta=1.4$ and $\mathrm{A}-\mathrm{TEM}_{\mathrm{p} 1}$ * beam consists both lower and higher order modes while $p=0,1,2,3,4,5$ and 6 , respectively. The fundamental lower order, a single-ringshaped azimuthally polarized $\mathrm{A}-\mathrm{TEM}_{01} *$ mode beam generates a focal hole in the focal point under the high NA focusing condition with relative electric field intensity distribution is shown in Fig. 3c. Figure 3a, b confirms that the DOF and FWHM of the generated focal hole segment is 1.86 and $0.368 \lambda$, respectively. The focal depth is measured at $r=0.35 \lambda$.

The higher order azimuthally polarized $\mathrm{A}-\mathrm{TEM}_{\mathrm{p} 1}$ * mode beams generates both uniform and non-uniform focal structures in the focal point under the high NA focusing circumstance. Once $p=1$, tight focusing of incident a single-ring-shaped azimuthally polarized beam became a double-ring-shaped azimuthally polarized beam as a basic mode in higher order. The electric field intensity distribution of the double-ring-shaped azimuthally polarized $\mathrm{A}-\mathrm{TEM}_{11}$ * mode beam calculated numerically and is shown in Fig. 4a. It is observed that the generated homogeneous focal hole have the focal depth is $3.4 \lambda$ with measured FWHM of the focal ring is around $0.342 \lambda$. By utilizing the A-TEM $\mathrm{A}_{11}$ * mode could generated an optical flat top beam in the focal region and it is used as an enormous tool for particle manipulation.

We further increased the mode number as $p=2$, which corresponds to triple-ring-shaped azimuthally polarized 

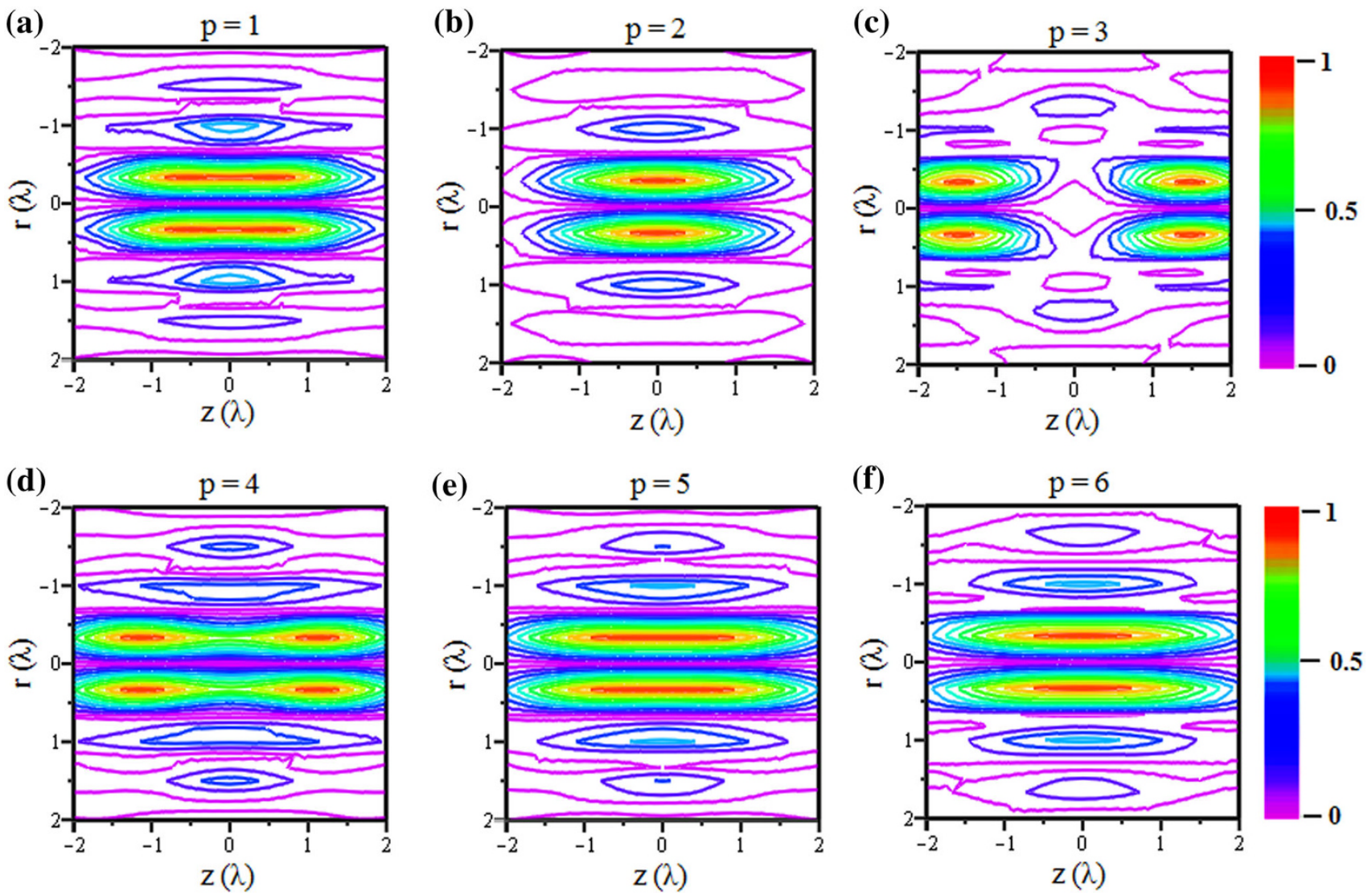

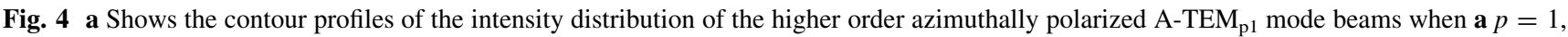
$\mathbf{b} p=2, \mathbf{c} p=3, \mathbf{d} p=4$ e $p=5$ and $\mathbf{f} p=6$ focused by high NA lens

A-TEM ${ }_{21} *$ mode beam. It is observed from Fig. 4b, the generated focal hole segment is uniform but the FWHM gradually increased as $0.356 \lambda$ and focal depth decreased $2.52 \lambda$, compared to the previous case. Further, we increase the mode values as $p=3$ and 4; they generated non-uniform focal hole structures near the focus with small changes in the FWHM and DOF of the produced focal holes compared to the previous cases below the high NA focusing condition as shown in Fig. 4c, d.

Figure $4 \mathrm{e}$ demonstrates the uniform electric field intensity distribution of the six-ring-shaped azimuthally polarized beam with high NA objective lens for one of the higher order mode $p=5\left(\mathrm{~A}-\mathrm{TEM}_{51} *\right)$. In this case, the FWHM of the generated focal hole is reduced as $0.324 \lambda$, but its DOF is much increased in contrast to the earlier cases as $3.72 \lambda$. As a result, A-TEM ${ }_{51} *$ mode could generate a small at the same time long optical flat top beam in the focal point which be utilized as a great implement for particle manipulation. If further we increase the mode value as $p=6$, it generates a homogenous focal hole however with a decreased DOF as $3 \lambda$ at the same time increases the FWHM as $0.356 \lambda$ when compared to the previous case and is shown in Fig. 4f. So we concluded that while changing the modes from lower order into higher order, the FWHM and DOF of generated focal holes are drastically increased or gradually decreased considerably under the high NA focusing circumstance.

In order to compare the results of lower and the higher order mode beams, we neglected lower order A-TEM ${ }_{01}$ * mode beam and higher order modes with $p=3$ and 4 since they are found to generate bigger hole size and small DOF of non-uniform focal structures at the focal point. The modes such as $p=1,2,5$ and 6 are chosen and the intensity distributions in focal region for NA $=0.95$ is calculated. The FWHM and DOF are also calculated and are shown in Fig. 5a, b respectively. From both figures, the dotted black line shows the intensity distribution of higher order azimuthally polarized A-TEM ${ }_{61} *$ mode beam, the solid red 

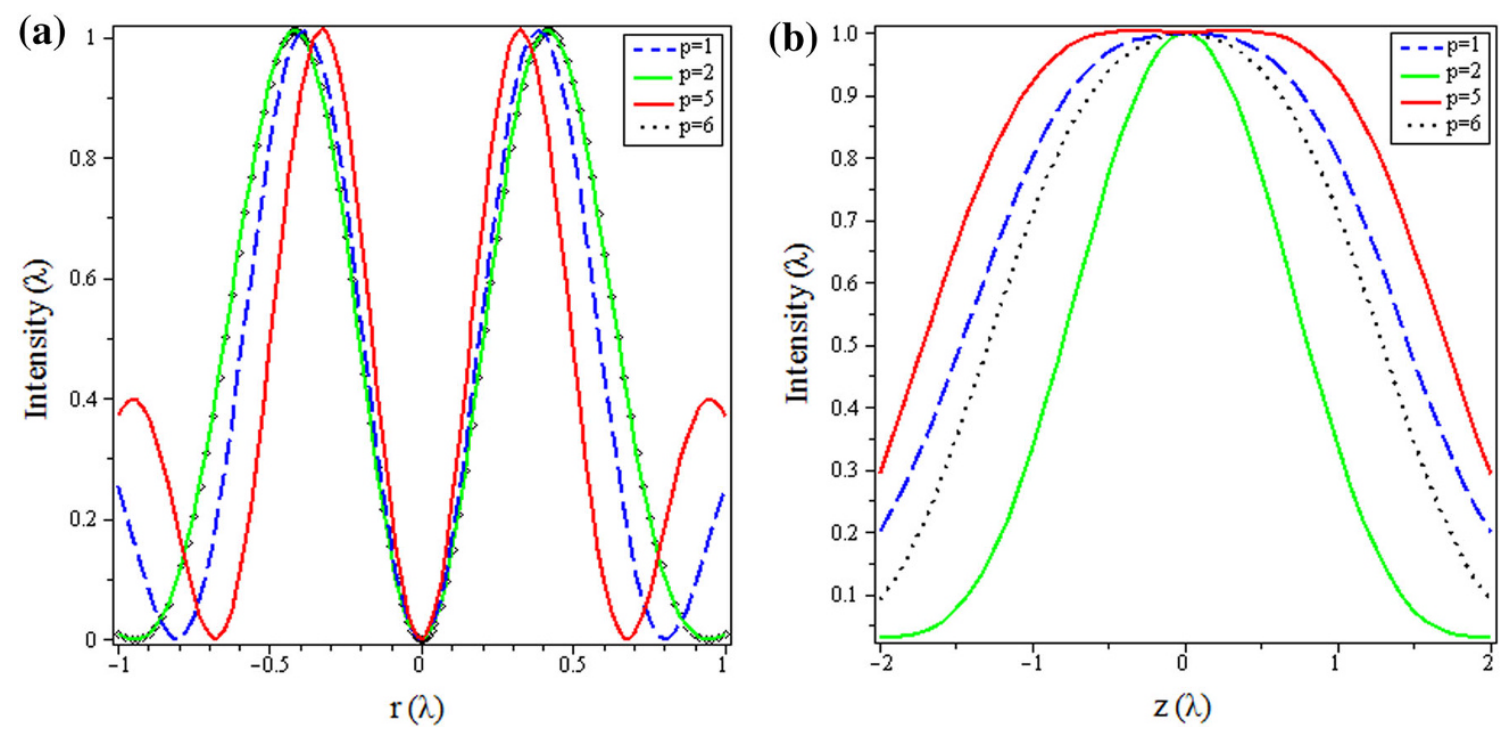

Fig. 5 Normalized intensity plots for a FWHM and b DOF for different higher order modes for (dashed blue line) A-TEM ${ }_{11}^{*}$, (solid green line) $\mathrm{A}-\mathrm{TEM}_{21} *$, (solid red line) A-TEM ${ }_{51} *$ and (dotted black line) A-TEM 61 of azimuthally polarized beams focused by high NA lens

line shows the intensity distribution of $\mathrm{A}^{-\mathrm{TEM}_{51}} *$ mode beam, solid green line shows the intensity distribution of A-TEM 21 mode beam and the dashed blue line shows the intensity distribution of $\mathrm{A}-\mathrm{TEM}_{11}$ * mode beam with high NA lens along transverse $(z=0 \lambda)$ and axial direction $(r=0.45 \lambda)$ for NA $=0.95$, respectively. The FWHM of the A-TEM ${ }_{51}$ * mode (solid red line) as shown in Fig. 4a is approximately $0.324 \lambda$, which is smaller than that achieved by using other higher order modes as $p=1,2$ and 6 (dashed blue, solid green and dotted black lines) with high NA lens system. Accomplished broadly utilized axially flat top beam by suggested mode $p=5$ (solid red line), which is extended compare to other higher order modes $p=1,2$ and 6 with high NA lens system (dashed blue, solid green and dotted black lines). It is clear that one of the extraordinary case of A-TEM ${ }_{51} *$ mode azimuthal polarized illumination gives better results compared to the above-mentioned all cases as well as lower order mode $\mathrm{A}-\mathrm{TEM}_{01}$ *.

Now, Fig. 6a, b shows the variations of FWHM and DOF of chosen higher order azimuthally polarized mode beams for different $\beta$ values (from 1.1 to 2 ) with $\mathrm{NA}=0.95$. Though tuning $\beta$ from 1.1 to 2 , the related values of FWHM and DOF are tabulated in Tables 1 and 2 for chosen higher order modes as $p=1,2,5$ and 6 . The FWHM of the ring focus decreases as well as increases on increasing $\beta$ incessantly as shown in Fig. 6a for chosen higher order modes. Conversely, the entire focal pattern progress as shown in Fig. $6 \mathrm{~b}$ is dissimilar and also we can notice that the focal hole expands under the condition of higher order modes for tuning $\beta$ values from 1.1 to 2 . Thus, $\beta$ affects the focal patterns noticeably. When the parameter $\beta$ increases from 1.1 to 2 , the focal pattern changes very surprisingly and most of the focal patterns have non-uniform focal structures under the focusing condition of $\mathrm{NA}=0.95$.

For example, we consider the parameter $\beta$ increasing from 1.1 up to 2 for the extraordinary mode $p=5$ (owing to small hole size with long DOF), the focal patterns were calculated and shown in Fig. 7. We can see from Fig. 7a that there are two intensity peaks on optical axis for $\beta=1.1$ as well as it has non-uniform focal structure. On increasing $\beta$ as 1.3 , the two intensity peaks broaden in transverse direction and the two dark peaks in geometrical focal plane minimizes into one dark hollow ring when tune $\beta=1.5$, as shown in Fig. $7 \mathrm{~b}$, c. After $\beta$ maintains to increase as $1.7,1.8$ and 2 , the intensity on optical axis increases significantly, so that ultimately two dark focal holes develops into one dark line along the optical axis and the intensity ring in geometrical focal plane finds stronger simultaneously, as shown in Fig. 7d-f. Therefore, one can attain a focal hole for different modes by tuning the pupil to beam ratio $(\beta)$ of the input azimuthally polarized beam. 

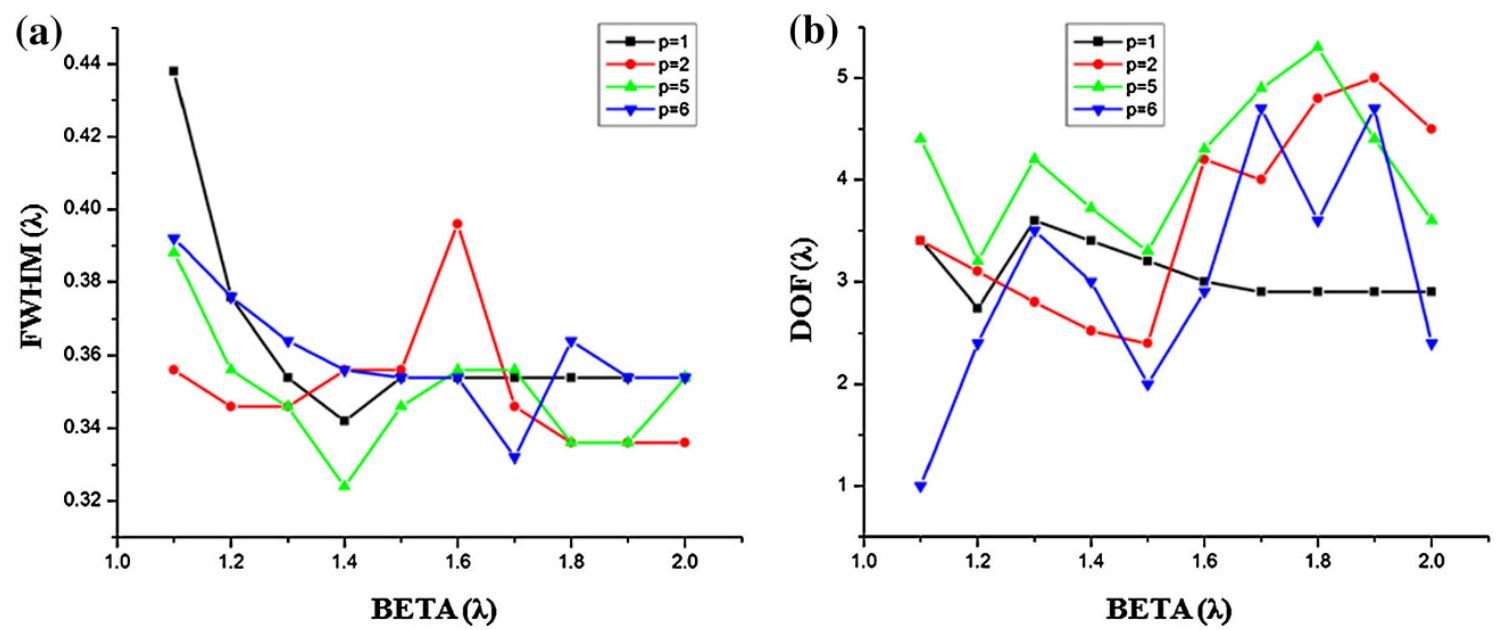

Fig. $6 \beta$ variations of a FWHM and b DOF for higher order modes as $p=1,2,5$ and 6

Table $1 \beta$ variations with modes $p=1,2$ with corresponding FWHM and DOF for NA $=0.95$

\begin{tabular}{|c|c|c|c|c|c|c|c|}
\hline \multirow{2}{*}{$\begin{array}{l}\text { S. } \\
\text { no. }\end{array}$} & \multirow{2}{*}{$\beta$} & \multicolumn{3}{|l|}{$p=1$} & \multicolumn{3}{|l|}{$p=2$} \\
\hline & & FWHM & DOF & Structure & FWHM & DOF & Structure \\
\hline 1 & 1.1 & 0.438 & 3.4 & $\begin{array}{l}\text { Non- } \\
\text { uniform }\end{array}$ & 0.356 & 3.4 & $\begin{array}{l}\text { Non- } \\
\text { uniform }\end{array}$ \\
\hline 2 & 1.2 & 0.376 & 2.74 & $\begin{array}{l}\text { Non- } \\
\text { uniform }\end{array}$ & 0.346 & 3.1 & Uniform \\
\hline 3 & 1.3 & 0.354 & 3.6 & $\begin{array}{l}\text { Non- } \\
\text { uniform }\end{array}$ & 0.346 & 2.8 & Uniform \\
\hline 4 & 1.4 & 0.342 & 3.4 & Uniform & 0.356 & 2.52 & Uniform \\
\hline 5 & 1.5 & 0.354 & 3.2 & Uniform & 0.356 & 2.4 & Uniform \\
\hline 6 & 1.6 & 0.354 & 3 & Uniform & 0.396 & 4.2 & $\begin{array}{l}\text { Non- } \\
\text { uniform }\end{array}$ \\
\hline 7 & 1.7 & 0.354 & 2.9 & Uniform & 0.346 & 4 & $\begin{array}{l}\text { Non- } \\
\text { uniform }\end{array}$ \\
\hline 8 & 1.8 & 0.354 & 2.9 & Uniform & 0.336 & 4.8 & $\begin{array}{l}\text { Non- } \\
\text { uniform }\end{array}$ \\
\hline 9 & 1.9 & 0.354 & 2.9 & Uniform & 0.336 & 5 & $\begin{array}{l}\text { Non- } \\
\text { uniform }\end{array}$ \\
\hline 10 & 2 & 0.354 & 2.9 & Uniform & 0.336 & 4.5 & $\begin{array}{l}\text { Non- } \\
\text { uniform }\end{array}$ \\
\hline
\end{tabular}

Finally, we consider only optical flat top tube beams in the focal region generated by $\mathrm{A}-\mathrm{TEM}_{11} *$ and $\mathrm{A}^{-\mathrm{TEM}_{51}}$ * modes in higher order azimuthally polarized beams for different high NA values. Figure 8a confirms that the FWHM of the optical flat top beams generated by $\mathrm{A}-\mathrm{TEM}_{51} *$ mode is much smaller while compared to the A-TEM 11 * mode and decreases homogeneously with the increments of NA. Figure $8 \mathrm{~b}$ illustrates that the DOF of the optical flat top tube beams generated by $\mathrm{A}-\mathrm{TEM}_{11}$ * and another extraordinary case of $\mathrm{A}-\mathrm{TEM}_{51} *$ modes are little unequal and diminish evenly with the increments of NA. In addition, it is seen from Table 3 that the maximum hole size along the axial direction and maximum DOF along the lateral direction for different NA values

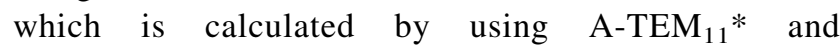
$\mathrm{A}-\mathrm{TEM}_{51} *$ modes in the ray optical rule. It is shown that both the DOF and the FWHM of the focal hole in the focus diminish with the increments of the numerical values of NA.

To evaluate the FWHM and DOF characteristics of higher and lower order mode beams, nevertheless, one of the higher order $\mathrm{A}-\mathrm{TEM}_{51}$ * mode beam generates an optical flat top tube beam encompass long focal depth $(3.72 \lambda)$ with reduced focal hole size $(0.324 \lambda)$ in the focal region. For future aspect, such kind of higher order mode beams which be appreciated by placing the diffractive optical element (DOE) on the lens pupil. This type of DOE (phase or amplitude filters) used to achieve long DOFs, maintain uniformity and reduced to beam size, respectively $[15,20]$. 
Table $2 \beta$ variations with modes $p=5,6$ and corresponding FWHM and DOF for $\mathrm{NA}=0.95$

\begin{tabular}{|c|c|c|c|c|c|c|c|}
\hline \multirow[t]{2}{*}{ S. no. } & \multirow[t]{2}{*}{ Beta } & \multicolumn{3}{|l|}{$\mathrm{p}=5$} & \multicolumn{3}{|l|}{$\mathrm{p}=6$} \\
\hline & & FWHM & DOF & Structure & FWHM & DOF & Structure \\
\hline 1 & 1.1 & 0.388 & 4.4 & Non-uniform & 0.392 & 1 & Non-uniform \\
\hline 2 & 1.2 & 0.356 & 3.2 & Non-uniform & 0.376 & 2.4 & Non-uniform \\
\hline 3 & 1.3 & 0.346 & 4.2 & Non-uniform & 0.364 & 3.5 & Uniform \\
\hline 4 & 1.4 & 0.324 & 3.72 & Uniform & 0.356 & 3 & Uniform \\
\hline 5 & 1.5 & 0.346 & 3.3 & Uniform & 0.354 & 2 & Non-uniform \\
\hline 6 & 1.6 & 0.356 & 4.3 & Non-uniform & 0.354 & 2.9 & Non-uniform \\
\hline 7 & 1.7 & 0.336 & 4.9 & Non-uniform & 0.332 & 4.7 & Non-uniform \\
\hline 8 & 1.8 & 0.336 & 5.3 & Non-uniform & 0.364 & 3.6 & Uniform \\
\hline 9 & 1.9 & 0.336 & 4.4 & Non-uniform & 0.354 & 4.7 & Non-uniform \\
\hline 10 & 2 & 0.354 & 3.6 & Uniform & 0.354 & 2.4 & Non-uniform \\
\hline
\end{tabular}

(a)

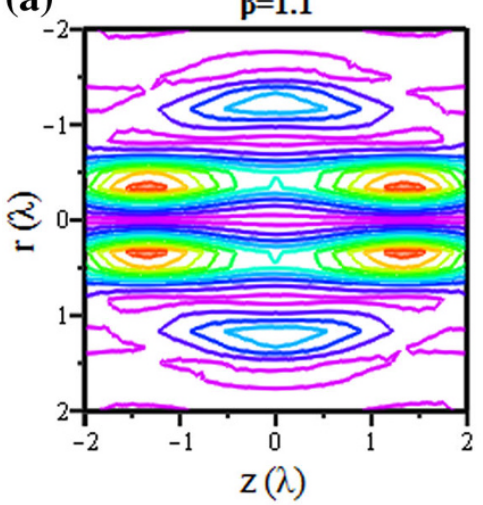

(d)

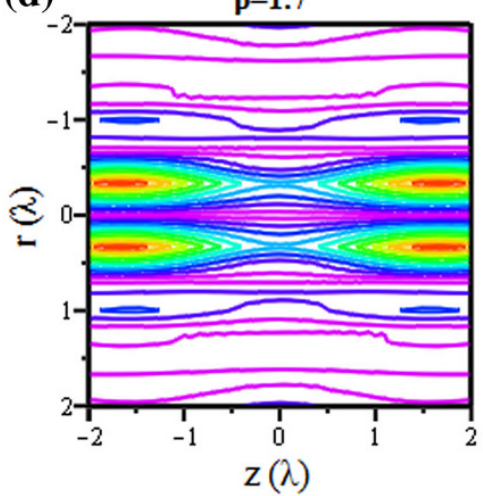

(b)

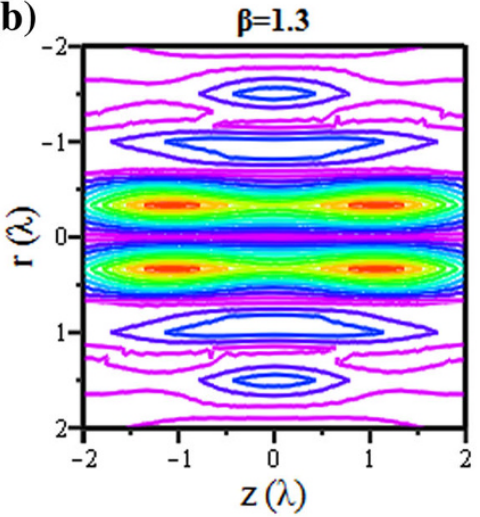

(e)

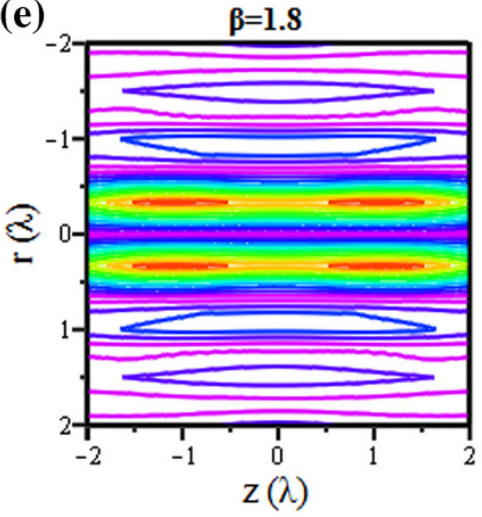

(c)

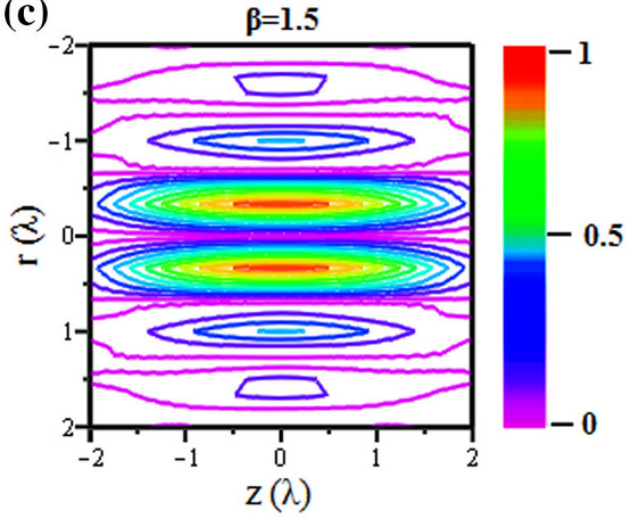

(f)

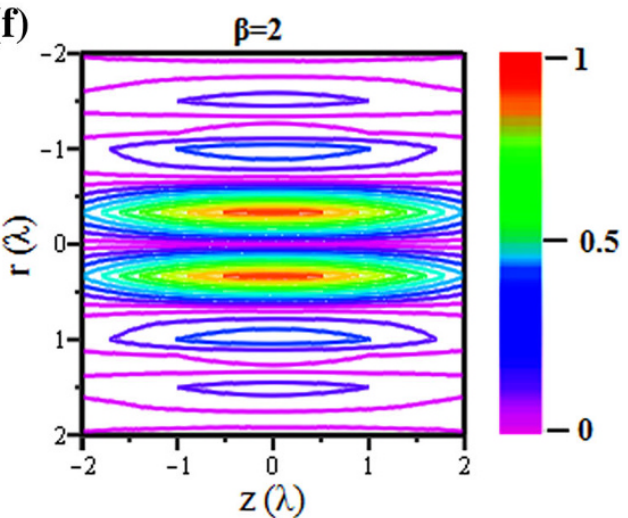

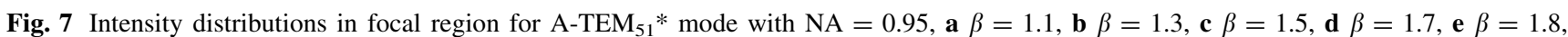
f $\beta=2$

\section{Conclusion}

In conclusion, we investigated the intensity distributions near the focal point for tight focusing of azimuthally polarized Laguerre-Gaussian beam with higher order modes are calculated based on vector diffraction theory. In a limit of $\mathrm{NA}=0.95$, the higher order $\mathrm{A}-\mathrm{TEM}_{\mathrm{p} 1}$ * mode beams of $p=1,2,3,4,5$ and 6 are established numerically in detail. One of the higher order mode, A-TEM ${ }_{51}{ }^{*}$, generated some interesting results and also 

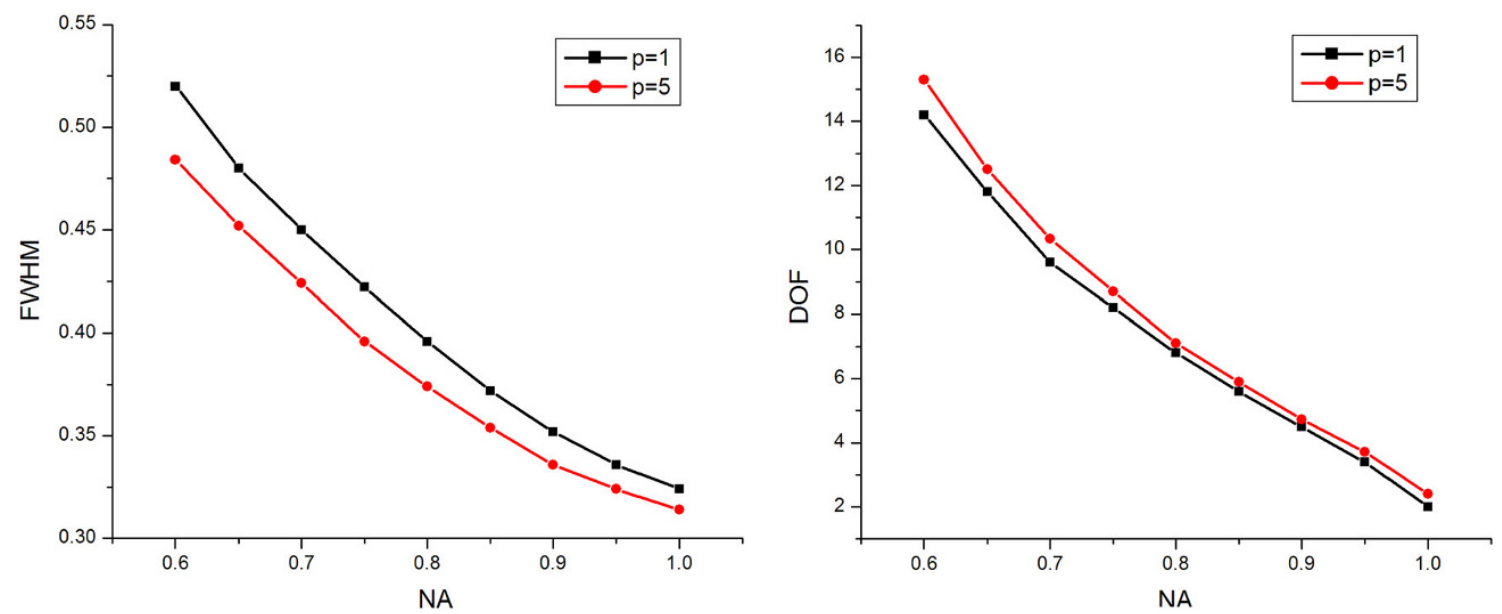

Fig. 8 The intensity distribution of a FWHM and $\mathbf{b}$ DOF of the focal hole generated by higher order mode $p=1$ and 5 for NA variations

Table 3 NA variations for modes $p=1,5$ with $\beta=1.4$ and corresponding FWHM and DOF

\begin{tabular}{|c|c|c|c|c|c|c|c|}
\hline \multirow[t]{2}{*}{ S. no. } & \multirow[t]{2}{*}{ NA } & \multicolumn{3}{|l|}{$p=1$} & \multicolumn{3}{|l|}{$p=5$} \\
\hline & & FWHM & DOF & Structure & FWHM & DOF & Structure \\
\hline 1 & 0.6 & 0.52 & 14.2 & Non-uniform & 0.484 & 15.3 & Non-uniform \\
\hline 2 & 0.65 & 0.48 & 11.8 & Non-uniform & 0.452 & 12.5 & Non-uniform \\
\hline 3 & 0.7 & 0.45 & 9.6 & Non-uniform & 0.424 & 10.34 & Non-uniform \\
\hline 4 & 0.75 & 0.422 & 8.2 & Non-uniform & 0.396 & 8.7 & Non-uniform \\
\hline 5 & 0.8 & 0.396 & 6.8 & Non-uniform & 0.374 & 7.1 & Non-uniform \\
\hline 6 & 0.85 & 0.372 & 5.6 & Non-uniform & 0.354 & 5.9 & Non-uniform \\
\hline 7 & 0.9 & 0.352 & 4.5 & Non-Uniform & 0.336 & 4.72 & Non-uniform \\
\hline 8 & 0.95 & 0.342 & 3.4 & Uniform & 0.324 & 3.72 & Uniform \\
\hline 9 & 1 & 0.324 & 2 & Uniform & 0.314 & 2.4 & Uniform \\
\hline
\end{tabular}

premeditated the intensity distribution of the different modes has little variation among the truncation coefficient $\beta$ of the input beam beside the pupil. This work is important for optical manipulation, optical data storage, micro-machines and optical trapping applications for low refractive index particles.

Acknowledgments One of the authors K. Lalithambigai expresses her sincere thanks to Basic Scientific Research (BSR), New Delhi, India [UGC Letter No. 11-142/2008(BSR)] for financial support.

Conflict of interest The authors declare that they have no competing interests.

Author contribution All authors provided the same contributions in this article. All authors have read and approved the final manuscript.

Open Access This article is distributed under the terms of the Creative Commons Attribution License which permits any use, distribution, and reproduction in any medium, provided the original author(s) and the source are credited.

\section{References}

1. Cizmar, T., Romero, L.C.D., Dholakia, K., Andrews, D.L.: Multiple optical trapping and binding: new routes to selfassembly. J. Phys. B 43, 102001 (2010)

2. Friedman, N., Kaplan, A., Davidson, N.: Dark optical traps for cold atoms. Adv. At. Mol. Opt. Phys. 48, 99-151 (2002)

3. Watanabe, T., Iketaki, Y., Omatsu, T., Yamamoto, K., Sakai, M., Fuji, M.: Two point separation in super-resolution fluorescence microscope based on up-conversion fluorescence depletion technique. Opt. Express 11, 3271-3276 (2003)

4. Torok, P., Munro, P.R.T.: The use of Gauss-Laguerre vector beams in STED microscopy. Opt. Express 12, 3605-3617 (2004)

5. Courtial, J., Padgett, M.J.: Limit to the orbital angular momentum per unit energy in a light beam that can be focused onto a small particle. Opt. Commun. 173, 269-274 (2000)

6. Ladavac, K., Grier, D.: Microoptomechanical pumps assembled and driven by holographic optical vortex arrays. Opt. Express 12, 1144-1149 (2004)

7. Jeffries, G.D.M., Edgar, J.S., Zhao, Y., Shelby, J.P., Fong, C., Chiu, D.T.: Using polarization-shaped optical vortex traps for single-cell nanosurgery. Nano Lett. 7, 415-420 (2007) 
8. Gahagan, K.T., Swartzlander, G.A.: Simultaneous trapping of low-index and high-index microparticles observed with an optical-vortex trap. J. Opt. Soc. Am. B 16, 533-537 (1999)

9. Gahagan, K.T., Schwartzlander, G.A.: Optical vortex trapping of particles. Opt. Lett. 21, 827-829 (1996)

10. Friese, M.E.J., Nieminen, T.A., Heckenberg, N.R., RubinszteinDunlop, H.: Optical alignment and spinning of laser-trapped microscopic particles. Nature 394, 348-350 (1998)

11. Cao, J., Chen, Q., Guo, H.: Creation of a controllable three dimensional optical chain by TEM01 mode radially polarized Laguerre-Gaussian beam. Optik 124, 2033-2036 (2013)

12. Moser, T., Glur, H., Romano, V., Pigeon, F., Parriaux, O., Ahmed, M.A., Graf, T.: Polarization- selective grating mirrors used in the generation of radial polarization. Appl. Phys. B 80, 707-713 (2005)

13. Zhuang, Y., Zhang, Y., Ding, B., Suyama, T.: Trapping Rayleigh particles using highly focused higher-order radially polarized beams. Opt. Commun. 284, 1734-1739 (2011)

14. Kozawa, Y., Sato, S.: Focusing property of a double-ring-shaped radially polarized beam. Opt. Lett. 31, 820-822 (2006)
15. Guo, H., Weng, X., Jiang, M., Zhao, Y., Sui, G., Hu, Q., Wang, Y., Zhuang, S.: Tight focusing of a higher-order radially polarized beam transmitting through multi-zone binary phase pupil filters. Opt. Express 21, 5363-5372 (2013)

16. Richards, B., Wolf, E.: Electromagnetic diffraction in optical systems, II. Structure of the image field in an aplanatic system. Proc. R. Soc. Lond. A Math. Phys. Sci. 253, 358-379 (1959)

17. Youngworth, K.S., Brown, T.G.: Focusing of high numerical aperture cylindrical-vector beams. Opt. Express 7, 77-87 (2000)

18. Lalithambigai, K., Saraswathi, R.C., Anbarasan, P.M., Rajesh, K.B., Jaroszewicz, Z.: Generation of multiple focal hole segments using double-ring shaped azimuthally polarized beam. J. Atomic Mol. Phy. 2013, 1-4 (2013)

19. Rajesh, K.B., Jaroszewicz, Z., Anbarasan, P.M.: Improvement of lens axicon's performance for longitudinally polarized beam generation by adding a dedicated phase transmittance. Opt. Express 18, 26799-26805 (2010)

20. Lin, J., Yin, K., Li, Y.D., Tan, J.B.: Achievement of longitudinally polarized focusing with long focal depth by amplitude modulation. Opt. Lett. 36, 1185-1187 (2011) 